\title{
Does dual task training improve walking performance of older adults with concern of falling?
}

\author{
B. Wollesen ${ }^{1 *}$ D, S. Schulz ${ }^{1}$ L. Seydell ${ }^{1}$ and K. Delbaere ${ }^{2}$
}

\begin{abstract}
Background: Older adults with concerns of falling show decrements of gait stability under single (ST) and dual task (DT) conditions.

To compare the effects of a DT training integrating task managing strategies for independent living older adults with and without concern about falling (COF) to a non-training control group on walking performance under ST and DT conditions.

Methods: Single center parallel group single blind randomized controlled trial with group-based interventions (DT-managing balance training) compared to a control group (Ninety-five independent living older adults; $71.5 \pm 5.2$ years).

A progressive DT training (12 sessions; 60 min each; 12 weeks) including task-managing strategies was compared to a non-training control group. Setting: group based intervention for independent living elderly in a gym. ST and DT walking (visual verbal Stroop task) were measured on a treadmill. Gait parameters (step length, step width, and gait line) and cognitive performance while walking were compared with a 2×2×2 Repeated Measures Analyses of Variance.
\end{abstract}

Results: Participants in the intervention group showed an increased step length under ST and DT conditions following the intervention, for both people with and without CoF compared to their respective control groups. Foot rolling movement and cognitive performance while walking however only improved in participants without CoF.

Conclusions: The results showed that DT managing training can improve walking performance under ST and DT conditions in people with and without CoF. Additional treatment to directly address CoF, such as cognitive behavioural therapy, should be considered to further improve the cautious gait pattern (as evidenced by reduced foot rolling movements).

Trial registration: The study was retrospectively registered in the German Clinical Trials Register (DRKS; Identification number DRKS00012382, 11.05.2017).

Keywords: Physical exercise, Fear of falling, Gait, Accidental falls

\section{Background}

Falls pose a major threat to the well-being and quality of life of older people. Falls can result in fractures and other injuries, disability and concern about falling (CoF), which trigger a decline in physical function and walking performance [1-3] and increase an individual's risk of future falls $[4,5]$. CoF is very common for independent-

\footnotetext{
* Correspondence: bettina.wollesen@uni-hamburg.de

${ }^{1}$ Human Movement Science, University of Hamburg, Mollerstr. 10, Turmweg,

20148 Hamburg, Germany

Full list of author information is available at the end of the article
}

living older adults, with prevalence rates often exceeding those of falls themselves [6]. About half of older adults express some level of CoF, and women are more commonly affected than men [7].

Previous research [8] has demonstrated that CoF can induce gait adaptations, by manipulating the environment in a way that exacerbates the potential consequences of a fall. These experimental studies have suggested that CoF decreases walking speed and step length, and increases double support time. It is now well-accepted that walking is not just a rhythmic and automated process, but also 
demands attention [9]. These demands increase with age, and with the complexity of the task being performed. More specifically, the ability to inhibit inappropriate responses and selectively attend to relevant environmental features while suppressing other inputs [10]. Since most daily life activities include some level of dual-tasking, these executive functioning skills (i.e. inhibitory skills) are required. It has been proposed that people with higher levels of $\mathrm{CoF}$ cannot inhibit or ignore irrelevant information of the environment in the process of balance control. Therefore, during the cognitive process the $\mathrm{CoF}$ competes for the limited resources of attentional focus to maintain balance control [11], resulting in an increased gait variability, instability and fall risk. For single task (ST) walking performance, a meta-analysis by Ayoubi et al. [12] revealed significant effects of $\mathrm{CoF}$ expressed in increased gait variability. Under DT conditions, a study by Donoghue et al. [13] found reduced gait speed and step length, especially for older persons who reduced their daily physical activity due to their CoF.

Systematic review evidence [14] has demonstrated that DT training studies can improve DT walking performance (cadence, gait variability, walking speed, foot rolling $[15,16])$. It has been suggested that better transfer effects can be achieved when DT training includes task managing strategies like task-switching training (switch of attention from one task to another [17]) or task prioritization training (focus on one of the tasks [18]). These aspects have not been integrated to intervention programs for older people with CoF, yet. Hence, it is not clear whether older adults with CoF benefit from this kind of DT training due to the additional cognitive efforts, which affect the attentional resources of this target group [10] in DT conditions. Therefore, the study aim was to examine whether a progressive DT balancetraining intervention with a focus on task managing strategies (task switching and task prioritization) could improve DT walking performance in older adults with and without CoF. The hypothesis is that DT training effects with task managing strategies are beneficial to those participants with $\mathrm{CoF}$ because they address the cognitive skills needed for walking and dual-task walking.

\section{Aim}

The primary aim of this study was to compare the effects of a DT training integrating task managing strategies for independent living older adults with and without concern about falling to a non-training control group on walking performance under ST and DT conditions.

\section{Methods}

The study was retrospectively registered in the German Clinical Trials Register (DRKS; Identification number DRKS00012382, 11.05.2017).

\section{Design}

A single blind randomized controlled trial investigated the effect of a DT group-based intervention on DT walking after 12 weeks. The study was approved by the ethics committee of the Hamburg Chamber of Physicians (registration number PV4376). All participants were informed about the study goals, and signed informed consent according to the Declaration of Helsinki. The setting was a group-based training (up to 15 persons in each training group) for independent elderly in a gym.

\section{Participants and recruitment}

A total number of 100 participants were recruited through advertisements in local newspapers. The inclusion criteria were: independent-living; age $65-80$ years, able to walk without a walking aid and capable of attending the group training. Exclusion criteria were: acute or chronical diseases with a documented influence on balance control (e.g. Parkinson's Disease; Diabetes Mellitus); cognitive impairment (Mini Mental Status Exam (MMSE) of less than 25); or participation in other exercise programs that could potentially confound the primary outcome. Figure 1 shows the study design and participant flow.

\section{Randomisation}

The randomisation sequence was determined using a webbased program (http://www.randomizer.org) and which was conducted by a person not involved in the study. Randomisation was stratified by the Short Physical Performance Battery (SPPB) [19], Falls Efficacy Scale international (FES-I) [20], sex and age ( \pm 2 years). Participants were randomized in a DT-managing balance training and a control group (cf. Fig. 1).

\section{Description of the intervention DT-training}

The group-based $D T$ training program was conducted for twelve weeks, during one $60 \mathrm{~min}$ session per week, including 8 to 15 participants per group. The group session was instructed by specialized DT trainers in a gymnasium [21]. The trainers were specialized for the regime of the DT training. They are physical therapist with additional background of human movement sciences or human movement scientists with additional licenses for older aged participants and falls prevention.

During the first 6 weeks, the program focused on daily situations which are commonly associated with an increased fall risk and included mostly challenging over ground walking exercises (brisk walking, start-stop exercises, walking with sidesteps, walking with turns, walking while negotiating tripping hazards [22]). To further increase difficulty levels, additional challenges were added to the walking exercises: faster speed, reduced visual input (e.g. looking up to the sky or closing their eyes), 


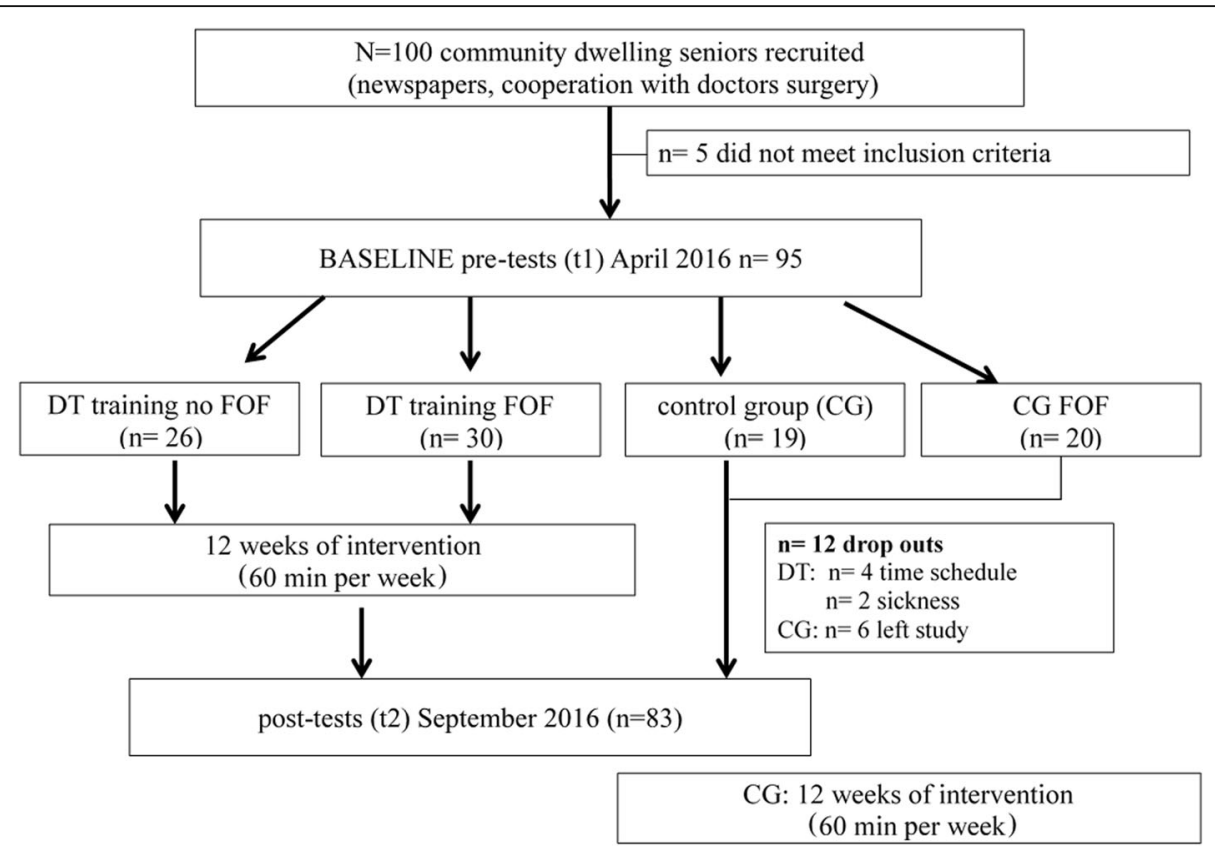

Fig. 1 shows the study design and participant flow

reduced proprioceptive input (e.g. walking on compliant surfaces), and reduced area of support. During these walking exercises, participants were also exposed to a variety of cognitive tasks, designed to challenge their focus of attention (e.g. reacting to signs for changing directions or following rhythms introduced by the instructor). In addition to physical and visual modifications (speed, visual input) task managing strategies targeting inhibitory skills and task prioritization were introduced (i.e., prioritization of the motor task, the cognitive task or both tasks at the same time).

During the last 6 weeks, the program focused more on task prioritization, task-switching and transfer into daily situations. The tasks became more complex and included visio-spatial and executive function tasks (e.g. reaction on signs that say turn right but the instruction was to turn left). Additionally, all tasks from the first phase were trained under DT conditions combined with precision tasks, time pressure, task prioritization and task switching. Daily situations were imitated (e.g. carrying shopping bags in a crowd of people while avoiding obstacles or reacting on signs while shifting around other people) to practice task managing strategies.

The instructors explained all exercises with additional verbal feedback to improve ask performance. The importance of each exercise was explained in the context of fall prevention (education, increasing knowledge).

The training protocol was standardized and could be repeated by all participant similarly.

\section{Control group}

The control group participants did not receive any exercises for twelve weeks and carried on with their usual activities.

\section{Primary outcome measure}

ST and DT walking performance was assessed during a 30-s walking test at self-selected constant speed on a treadmill (h/p/cosmos, Zebris; Isny, Germany: FDM-T). Before the test session the participants practiced treadmill walking for about five minutes in order to become familiar with the ST and DT walking task until they felt comfortable. A staircase method was used with going up to a certain level of comfortable walking speed and increasing and decreasing from that point until comfortable pace is achieved afterwards. Gait parameters such as step length $(\mathrm{cm})$, step width $(\mathrm{cm})$, and gait line, which describes the length of the foot rolling movements $(\mathrm{cm})$ were measured as main outcome parameters. Our training regime focused mainly on the gait quality and associated the kinematic parameters. Since there is a controversial discussion about gait variability, for this study we focused on the reported gait parameters, which have been shown to be important walking variables as well [21].

A visual-verbal Stroop test with 30 events of incongruent coloured words (e.g. the word "blue" presented in a yellow font) was added to the walking test as a cognitive DT. Stimuli were projected onto a white wall two meters in front of the participants. Participants were asked to name the colour of the font letters word and inhibit 
reading the word. The time interval between word insertions randomly varied between 0.8 and $1.2 \mathrm{~ms}$ to avoid rhythm. A randomized process distributed three out of four different versions of the Stroop test to the participants (1. familiarization while sitting, 2. ST standing, 3. DT combined with treadmill walking) were presented to the participants. All tests were recorded as a Stroop video including the verbal responses to the visualised colour word on the screen. The number of correct answers was analysed.

\section{Other measures}

Demographics, anthropometric data and comorbidities were assessed at baseline with a standardised questionnaire. Health-related quality of life was examined with the Short Form -12 questionnaire (SF 12 [23]).

$\mathrm{CoF}$ and fall risk were assessed as secondary outcome measures at baseline and after 12 weeks. The FES-I was used to examine concern about falling during 16 daily activities. The 16 items are rated as not at all concerned (1) to very concerned (4). Higher scores are indicative of greater concerns of falling. Participants with FES-I scores of 20 or higher were categorized into the higher $\mathrm{CoF}$ [20]. Intervention groups were stratified by the SPBB, due to previously established links between SPPB and kinematic parameters of walking $[13,21]$. The SPPB was used as a control variable for the randomization process based on tests of static balance, walking speed over $4 \mathrm{~m}$ (at normal pace), and the five times chair stand test. Each test score is categorized from 1 (worst) to 4 (best); the overall sum is then used to create a SPPB summary performance scale.

\section{Sample size}

A priori sample size calculation (G* power 3.1., ANOVA: Repeated measures within factors; $f=0.20$; alpha error probability $=0.05$; power $=0.80$; number of groups $=2 \times 2$; number of measurements $=2$ ) calculated a total number of 76 participants. With an anticipated dropout rate of $20 \%$, the recruitment aimed for 92 participants.

\section{Statistical analysis}

Twelve participants (six in the DT training group and six in the control group) did not have data available after 12 weeks (cf. Fig. 1). The main causes for drop out in the DT training group were problems with the time schedule (e.g. holidays $n=4)$ and illness $(n=2)$. Six participants of the control group left the study because of their group allocation. Repeated measures ANOVA were used to determine the intervention effect on the primary outcome at follow-up. Three-way (comparing $2 \times 2 \times 2$ groups) repeated measures ANOVAs were used for each outcome variable (e.g. step length, step width, FES-I). Analyses were performed with SPSS version 22 (IBM statistics Armonk, NY). Main effects for time (pre/post), group (intervention/control) and CoF (low $=$ FES- $I<20$ /high FES-I 20 and above) were reported, as well as between-subject effects for the groups (Group $\mathrm{x}$ time $\mathrm{x}$ CoF). Significance level was set at a two-sided $\alpha$ of 5\%; normal distribution was assessed using the KolmogorowSmirnow test. Variables were transformed as required to ensure statistical assumptions were met. Effect sizes are given as partial eta squares $\left(\eta_{\mathrm{p}}^{2}\right.$; small effect $\eta_{\mathrm{p}}^{2} \geq 0.08$, medium effect $\eta_{\mathrm{p}}^{2} \geq 0.20$, and $\eta_{\mathrm{p}}^{2} \geq 0.32$ large effect, [24]. Bonferroni correction was applied to post-hoc comparisons.

\section{Results}

Table 1 gives an overview of the main characteristics $(N=95)$ of the analysed participants.

No significant group differences were found for demographic, gender and health-related data.

\section{Main effect of the intervention on participants with and without CoF}

Table 2 shows all results of the three-way ANOVA. It shows significant effects of time - between pre to post measurements - for all examined gait parameters independent of the group allocation. The time $\times$ group interaction effects demonstrated a greater improvement for step length and gait line under ST and DT conditions of the intervention groups (with and without CoF). Post-hoc comparisons revealed an increased step length for both feet and gait line for the right foot (trend for left foot) in the intervention group $(p<0.001)$. In the DT conditions, there was also a time $\times$ group effect for the FES-I and DT walking test, as demonstrated by a reduced FES-I score $(p<0.001)$ and number of errors during the DT Stroop walking test in the intervention group $(p<0.05)$.

\section{Differentiating effect of the intervention in people with and without CoF}

There were no strong time $\times$ group $\times$ CoF effects for ST or DT walking, with only a significant effect for the right foot gait line under ST conditions (Table 2). Participants without $\mathrm{CoF}$ in the intervention group showed greater increases for the gait-line of the right foot $(p<0.05)$. The FES-I decreased in people with CoF who had the intervention compared to the control group, and remained unchanged in the no CoF group. The cognitive performance while DT walking improved in people in the intervention group without $\mathrm{CoF}$ compared to the control group, with no effect in people with $\mathrm{CoF}(p<0.05)$.

\section{Discussion}

The present study examined whether a progressive DT balance-training intervention with a focus on task managing strategies (task switching and task prioritization) 
Table 1 Mean (SD) or Number (\%) of the Groups for the Demographic Characteristics of $N=95$ Participants at baseline

\begin{tabular}{|c|c|c|c|c|}
\hline Characteristics & $\begin{array}{l}\text { Intervention with } \\
\text { FES- } l<20 \\
(n=26)\end{array}$ & $\begin{array}{l}\text { Intervention with } \\
\text { FES- }>20 \\
(n=30)\end{array}$ & $\begin{array}{l}\text { Control group with } \\
\text { FES-I }<20(n=19)\end{array}$ & $\begin{array}{l}\text { Control group with } \\
\text { FES-I > } 20(n=20)\end{array}$ \\
\hline Age (yr) & $72.2(4.6)$ & $69.8(5.7)$ & $72.9(4.4)$ & $72.7(5.3)$ \\
\hline Females, number (\%) & $16(61.5 \%)$ & $28(86.7 \%)$ & $12(63.2 \%)$ & $17(85 \%)$ \\
\hline Height $(\mathrm{cm})$ females & $162.9(5.4)$ & $165.7(6.6)$ & $162.5(9.6)$ & $164.2(5.6)$ \\
\hline Height $(\mathrm{cm})$ males & $177.4(9.7)$ & $178.1(3.0)$ & $182.8(5.5)$ & $171.5(6.2)$ \\
\hline Weight $(\mathrm{kg})$ females & $64.3(12.3)$ & $72.3(11.0)$ & $70.6(10.7)$ & $73.8(12.1)$ \\
\hline Weight $(\mathrm{kg})$ males & $85.3(15.7)$ & $84.3(13.7)$ & $88.5(5.2)$ & $79.0(20.1)$ \\
\hline BMl females $\left(\mathrm{kg} / \mathrm{m}^{2}\right)$ & $24.3(3.7)$ & $25.9(3.5)$ & $26.7(3.5)$ & $27.2(3.6)$ \\
\hline BMI males $\left(\mathrm{kg} / \mathrm{m}^{2}\right)$ & $27.4(2.9)$ & $26.1(4.2)$ & $26.5(1) 0$. & $28.8(2.5)$ \\
\hline SPPB (score out of 12) & $11.2(0.9)$ & $10.8(1.4)$ & $10.9(1.3)$ & $10.8(1.0)$ \\
\hline Walking speed (m/s) & $4.49(0.8)$ & $4.25(0.9)$ & $4.84(0.7)$ & $4.0(1.2)$ \\
\hline Physical Problems (number) & $2.4(2)$ & $2.5(2)$ & $2.5(2)$ & $3.1(3)$ \\
\hline Chronic diseases (number of participants) & 8 & 15 & 7 & 9 \\
\hline Medications (number of participants) & 16 & 19 & 9 & 13 \\
\hline FES-I (score out of 64) & $17.9(2.6)$ & $23.4(2.9)$ & $17.6(.9)$ & $23.8(3.4)$ \\
\hline SF 12 physical(Reference score age group $37.76 \pm 12.27$ ) & $46.84(8.5)$ & $47.27(9.1)$ & $47.80(9.1)$ & $48.41(9.2)$ \\
\hline SF12 mental (Reference score age group $50.24 \pm 10.81$ ) & $49.65(6.7)$ & $51.65(9.4)$ & $52.10(9.4)$ & $53.20(7.9)$ \\
\hline
\end{tabular}

BMI Body Mass Index, SPPB Short physical performance battery, FES-I Falls Efficacy Scale International, SF12 Short Form -12 questionnaire

could improve DT walking performance in older adults with and without CoF. The results showed a significantly improved ST and DT walking performance for the intervention group regardless of $\mathrm{CoF}$ after 12 weeks of training. Improved walking performance was demonstrated through an increased step length and gait line. These common kinematic walking variables suggest an active use of the ankle joint and roll movements, which is an important strategy to compensate for gait impairment and maintain postural control while walking [25]. Gait improvements were evident for the participants of the intervention independent of CoF under both ST and DT conditions, similar to previous studies using similar DT interventions with task-managing strategies [15-18, 21].

The overall aim of this present study was to explore whether there was a disparate effect of our DT intervention in older people with CoF. In line with previous studies [12, 13], participants with CoF walked slower at baseline with a reduced overall walking performance compared to people without CoF, under both ST and DT conditions. However, while our DT training, improved step length, equally in both groups independently of $\mathrm{CoF}$, our results show that the gait-line did not follow that same trend. This suggests that people with CoF still have more cautious gait pattern after the intervention with less foot rolling movements compared to the participants with no CoF [10, 26]. A recent Cochrane review has suggested that exercise can reduce $\mathrm{CoF}$ immediately following the intervention [27], however, there is less certainty about long-term effects. Our finding that foot rolling movement remain impaired following the intervention in people with CoF might be an explanation for a reduced effect of exercise for participants with CoF. Since the exercises did not address the $\mathrm{CoF}$, additional psychological support might be required to fully address CoF and the underlying reason for the reduced DT performance e.g. Cognitive Behaviour Training (CBT) for people with CoF.

Our results also show that cognitive performance while walking did not improve in people with $\mathrm{CoF}$, which also confirms that they might require additional support through strategies such as CBT to minimize the interference of $\mathrm{CoF}$ on the capacity to perform tasks requiring attentional resources. The resources allocation model by Kahnemann [28] suggests that a higher amount of attention is needed for motor and cognitive performance under DT conditions to manage both tasks similarly. If $\mathrm{CoF}$ competes for these attentional resources, walking will be more strongly affected by a DT in people with CoF. Following the task prioritization model by YogevSeligmann et al. [29] one might argue that the laboratory situation was not dangerous enough to switch from the cognitive focus to motor control. Therefore, future studies should include a more challenging walking task to control if this balance and task managing intervention is successful in a transfer to daily situations.

\section{Limitations}

We would like to acknowledge some limitations regarding the study design. Despite our attempts to stratify 


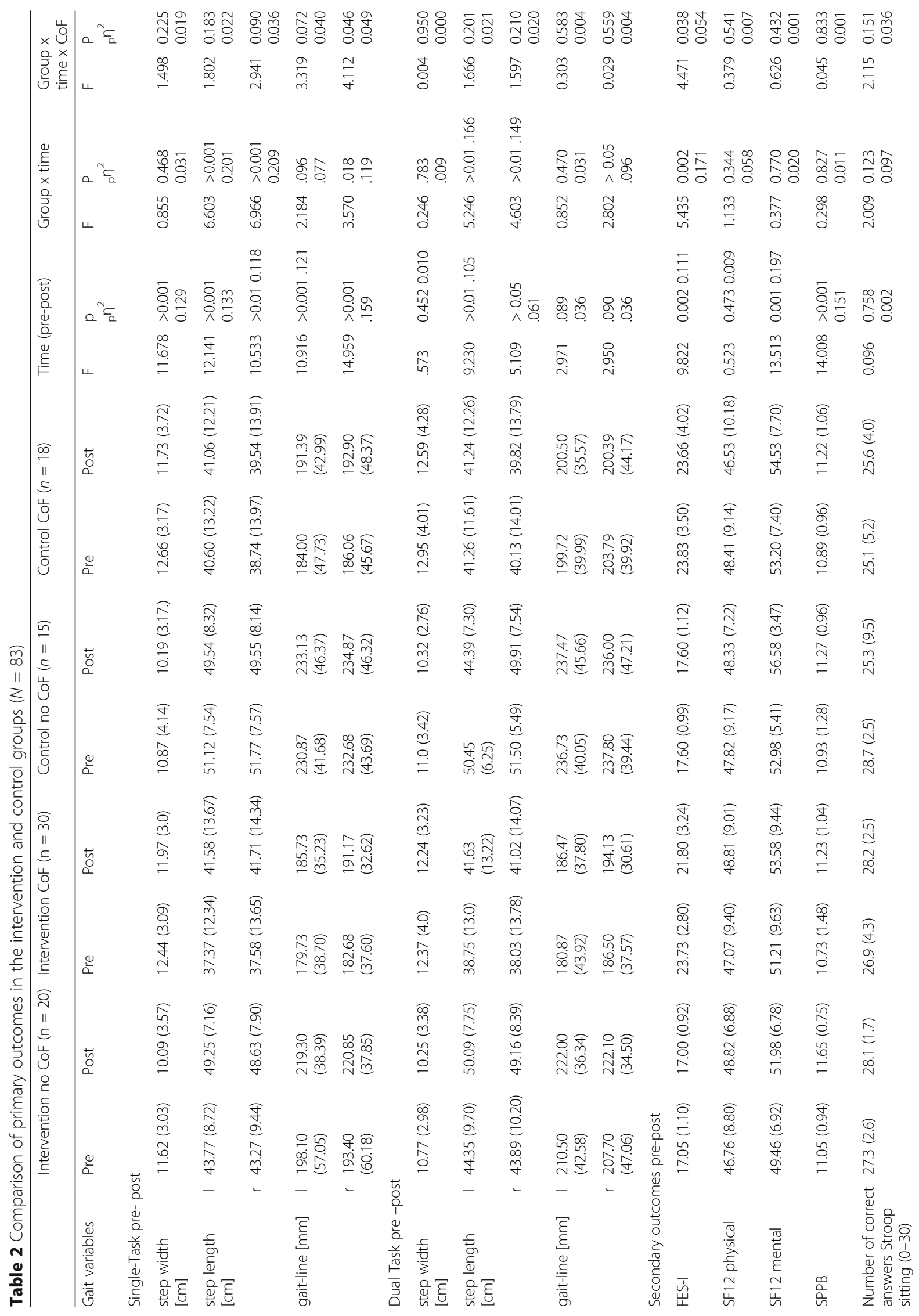




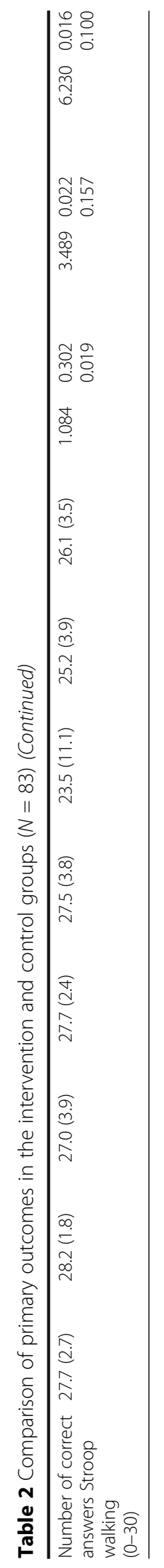


participants based on fall risk and COF into control and intervention, our groups were not equal. The control group participants had a higher walking speed and increased step length. Furthermore, participants were allocated using a 2:1 ratio to intervention and control groups, respectively. This decision was guided by our previous experiences that participants are often displeased by a control group allocation. This combined with a high attrition rate in the control group due to group allocation, amplified the differences in group size further for the effectiveness analyses, which might have affected the representativeness of the sample. Moreover, we did not include a pre-post fall risk assessment or an over-ground gait assessment. The study was not powered to look at differential effects of certain tasks or dual-task cost. Additionally, one might argue that the participants trained over ground walking performance, whereas the test situation was done on a treadmill. To avoid confounding factors, we followed the recommendations by Wollesen, Rönnfeldt and Mattes [30]. However, there is some evidence that there are no kinetic and kinematic differences when over ground walking is compared to treadmill walking [31, 32]. Nevertheless, the measurement on the treadmill with fixed selfselected walking speed does not allow the analysis of improvements in walking speed. In addition, due to the small sample size dual-task costs could not be analysed sufficiently. Therefore, these aspects should be controlled in future studies.

\section{Conclusions}

In conclusion, the present study demonstrated that our DT training intervention including task managing strategies was effective for both participants with and without CoF, by improving overall gait performance under ST and DT conditions. However, there was a disparate effect for people with CoF compared to people without CoF for the active foot rolling movements and cognitive performance while walking, where people with $\mathrm{CoF}$ showed less improvement following the intervention on both factors. To see long-term effects on CoF and gait performance, we suggest the addition of psychological strategies, such as CBT to DT training.

\section{Abbreviations \\ CBT: Cognitive Behaviour Training; CoF: Concerns of Falling: DT: Dual task: FES-I: Falls Efficacy Scale international; MMSE: Mini Mental Status Exam; SF 12: Short Form (12 questionnaire) SF 36 Health Survey; SPPB: Short Physical Performance Battery; ST: Single task}

\section{Acknowledgements}

The authors wish to thank the Department of Human Movement Science of the University of Hamburg and the Medical Centrum Hammoniabad for providing the intervention for participants living in the local area.

\section{Funding}

This study was conducted without external funding.
Availability of data and materials

Data can be obtained from the corresponding author upon reasonable request.

\section{Authors' contributions}

BW and KD had the study idea and conducted the methods. SS collected the data and helped with the data analysis. LS was responsible for the group training sessions. BW did the data analyses and wrote the manuscript. KD edited the manuscript. All authors have read and approved the manuscript.

\section{Ethics approval and consent to participate}

The study was approved by the ethics committee of the Hamburg Chamber of Physicians (registration number PV4376). All participants were informed about the study goals, and signed informed consent according to the Declaration of Helsinki.

\section{Consent for publication}

The manuscript did not contain any individual person's data in any form.

\section{Competing interests}

KD is an Associate Editor for BMC Geriatrics since 2015. There are no other financial or non-financial competing interests.

\section{Publisher's Note}

Springer Nature remains neutral with regard to jurisdictional claims in published maps and institutional affiliations.

\section{Author details}

${ }^{1}$ Human Movement Science, University of Hamburg, Mollerstr. 10, Turmweg, 20148 Hamburg, Germany. ${ }^{2}$ Department of Health Science, Neuroscience Research Australia, University of New South Wales, Margarete Ainsworth Building, Barker Street, Randwick, Sydney, NSW 2031, Australia.

Received: 12 May 2017 Accepted: 6 September 2017

Published online: 11 September 2017

References

1. Yardley L, Smith $H$. A prospective study of the relationship between feared consequences of falling and avoidance of activity in community-living older people. The Gerontologist. 2002;42(1):17-23. https:/doi.org/10.1093/geront/42.1.17

2. Reelick MF, van lersel MB, Kessels RPC, Olde Rikkert MGM. The influence of fear of falling on gait and balance in older people. Age Ageing. 2009;38(4): 435-40. https://doi.org/10.1093/ageing/afp066.

3. de Busch TA, Duarte YA, Pires Nunes D, Lebrão ML, Satya Naslavsky M, dos Santos Rodrigues A, et al. Factors associated with lower gait speed among the elderly living in a developing country: a cross-sectional population-based study. BMC Geriatrics. 2015;15:35. https://doi.org/10.1186/s12877-015-0031-2.

4. Zijlstra GAR, Van Haastregt JCM, Van Rossum E, Van Eijk JTM, Yardley L, Kempen GIJM. Interventions to reduce fear of falling in community-living older people: a systematic review. J Am Geriatr Soc. 2007;55(4):603-15. https://doi.org/10.1111/j.1532-5415.2007.01148.

5. Anders J, Dapp U, Laub S, Wolfgang Von Renteln-Kruse AH. Einfluss von Sturzgefährdung und Sturzangst auf die Mobilität selbstständig lebender, älterer Menschen am Übergang zur Gebrechlichkeit Screeningergebnisse zur kommunalen Sturzprävention [Influence of fall risk and fear of falling on the mobility of independently living, older people at the transition to the frailty of screening results to the local falls prevention]. Z Gerontol Geriat. 2007:4(40):255-267. https://doi.org/10.1007/s00391-007-0473-z.

6. Rochat S, Büla CJ, Martin E, Seematter-Bagnoud L, Karmaniola A, Aminian K, et al. What is the relationship between fear of falling and gait in wellfunctioning older persons aged 65 to 70 years? Arch Phys Med Rehabil. 2010;91(6):879-84

7. Arfken CL, Lach HW, Birge SJ, Miller JP. The prevalence and correlates of fear of falling in elderly persons living in the community. Am J Pub Health. 1994; 84(4):565-70

8. Delbaere K, Sturnieks DL, Crombez G, Lord SR. Concern about falls elicits changes in gait parameters in conditions of postural threat in older people. J Gerontol A Biol Sci Med Sci. 2009;64(2):237-42.

9. Yogev-Seligmann G, Hausdorff JM, Giladi N. The role of executive function and attention in gait. Mov Disord. 2008;23(3):329-42. 
10. Young WR, Williams AM. How fear of falling can increase fall-risk in older adults: applying psychological theory to practical observations. Gait Posture. 2015;41(1):7-12.

11. Lacour M, Bernard-Demanze L, Dumitrescu M. Posture control, aging, and attention resources: models and posture-analysis methods. Neurophysiol Clin. 2008;38(6):411-21.

12. Ayoubi F, Launay CP, Annweiler C, Beauchet O. Fear of falling and gait variability in older adults: a systematic review and meta-analysis. J Am Med Dir Assoc. 2015;16(1):14.

13. Donoghue OA, Cronin H, Savva GM, O'Regan C, Kenny RA. Effects of fear of falling and activity restriction on normal and dual task walking in community dwelling older adults. Gait Posture. 2013;38(1):120-4.

14. Wollesen $B$, Voelcker-Rehage $C$. Training effects on motor-cognitive dualtask performance in older adults. Eur Rev Aging Phys Act. 2013;11(1):5-24.

15. Silsupadol $P$, Lugade $V$, Shumway-Cook A, van Donkelaar $P$, Chou, et al. Training-related changes in dual-task walking performance of elderly persons with balance impairment: A double-blind, randomized controlled trial. Gait Posture. 2009;29:634-9.

16. Silsupadol P, Shumway-Cook A, Lugade V, van Donkelaar P, Chou LS, Mayr $U$, et al. Effects of single-task versus dual-task training on balance performance in older adults: a double-blind, randomized controlled trial. Arch Phys Med Rehabil. 2009;90:381-7.

17. Bherer L, Kramer AF, Peterson MS, Colcombe S. Erickson, Becic E. Transfer effects in task-set cost and dual-task cost after dual-task training in older and younger adults: further evidence for cognitive plasticity in attentional control in late adulthood. Exp Aging Res. 2008;34(3):188-219.

18. Li KZ, Roudaia E, Lussier M, Bherer L, Leroux A, Kinley PA. Benefits of cognitive dual-task training on balance performance in healthy older adults. J Gerontol A Biol Sci Med Sci. 2010;65(12):1344-52.

19. Guralnik JM, Simonsick EM, Ferrucci L, Glynn RJ, Berkman LF, Blazer DG, et al. A short physical performance battery assessing lower extremity function: association with self-reported disability and prediction of mortality and nursing home admission. J Gerontol. 1994;49:M85-94.

20. Delbaere K, Close JC, Mikolaizak AS, Sachdev PS, Brodaty H, Lord SR. The falls efficacy scale international (FES-I). A comprehensive longitudinal validation study. Age Ageing. 2010;39(2):210-6.

21. Wollesen B, Voelcker-Rehage C, Willer J, Zech A, Mattes K. Feasibility study of dual-task-managing training to improve gait performance of older adults. Aging Clin Exp Res. 2015;27(4):447-55. https:/doi.org/10.1007/s40520-014-0301-4

22. Hollman JH, Kovash FM, Kubik JJ, Linbo RA. Age-related differences in spatiotemporal markers of gait stability during dual task walking. Gait Posture. 2007;26(1):113-9.

23. Bullinger M, Kirchberger I. SF 36 Fragebogen zum Gesundheitszustand [SF 36 Health Survey] Handanweisung. Goettingen: Hogrefe; 1998.

24. Cohen J. Statistical power analysis for behavior sciences, 1988

25. Perry J. Gait analysis: normal and Pathodological function 2. SLACK Inc: Ed. NJ; 2010.

26. Al-Yahya E, Dawes H, Smith L, Dennis A, Howells K, Cockburn J. Cognitive motor interference while walking: a systematic review and meta-analysis. Neurosci Biobehav Rev. 2010;35:715-28.

27. Kumar A, Delbaere K, Zijlstra GAR, et al. Exercise for reducing fear of falling in older people living in the community: Cochrane systematic review and meta-analysis. Age Ageing. 2016;45(3):345-52.

28. Kahnemann D. Attention and effort. Englewood Cliffs: Prentice Hall; 1973.

29. Yogev-Seligmann G, Hausdorff JM, Giladi N. Do we always prioritize balance when walking? Towards an integrated model of task prioritization. Mov Disord. 2012;27:765-70.

30. Wollesen, B., Mattes, K., \& Rönnfeldt, J. (2016). Influence of age, gender and test conditions on the reproducibility of dual-task walking performance. Aging clinical and experimental research.

31. Beurskens R, Bock O. Does the walking task matter? Influence of different walking conditions on dual-task performances in young and older persons. Hum Mov Sci. 2013;32(6):1456-66.

32. Riley PO, Paolini G, Della C, et al. A kinematic and kinetic comparison of overground and treadmill walking in healthy subjects. Gait Posture. 2007;26: 17-24. doi:10.1016/j.gaitpost.2006.07.003.

\section{Submit your next manuscript to BioMed Central and we will help you at every step:}

- We accept pre-submission inquiries

- Our selector tool helps you to find the most relevant journal

- We provide round the clock customer support

- Convenient online submission

- Thorough peer review

- Inclusion in PubMed and all major indexing services

- Maximum visibility for your research

Submit your manuscript at www.biomedcentral.com/submit
Biomed Central 